\title{
Reduction of exercise-induced asthma in children by short, repeated warm ups
}

\author{
C de Bisschop, H Guenard, P Desnot, J Vergeret
}

\begin{abstract}
Aim-To study the effect of a warm up schedule on exercise-induced asthma in asthmatic children to enable them to engage in asthmogenic activities.

Method-In the first study, peak flows during and after three short, repeated warm up schedules (SRWU 1, 2, and 3), identical in form but differing in intensity, were compared in $\mathbf{1 6}$ asthmatic children. In the second study the efficiency of the best of these SRWU schedules was tested on 30 young asthmatic children. Children performed on different days a 7 minute run alone $(\mathrm{EX} 1)$ or the same run after an SRWU (EX2).

Results-The second study showed that for most children (24/30) the fall in peak flow after EX2 was less than that after EX1. The percentage fall in peak flow after EX2 was significantly correlated with the percentage change in peak flow induced by SRWU2 $(r=0.68)$. The children were divided into three subgroups according to the change in peak flow after SRWU2: (G1: increase in peak flow; G2: $<15 \%$ fall in peak flow; G3: > $15 \%$ fall in peak flow). Only the children in the G3 subgroup did not show any gain in peak flow after EX2 compared with EX1.

Conclusion-The alteration in peak flow at the end of the SRWU period was a good predictor of the occurrence of bronchoconstriction after EX2. An SRWU reduced the decrease in peak flow for most of the children (24/30) in this series, thus reducing subsequent post-exercise deep bronchoconstriction.

(Br F Sports Med 1999;33:100-104)
\end{abstract}

Keywords: bronchoconstriction; children; asthma; warm up

Réadaptation

Fonctionnelle

Respiratoire, 66760

Angoustrine

Villeneuve des

Escaldes, France

P Desnot

110 Avenue d'Arès, 33200 Bordeaux,

France

J Vergeret

Correspondence to:

C de Bisschop, Laboratoire

d'analyse de la performance

motrice humaine, Faculté

des Sciences du Sport, 4

allée Jean Monnet, 86000

Poitiers, France.

Accepted for publication 22 October 1998 short, repeated warm up periods (SRWU) have been shown to reduce the bronchospasm induced by a subsequent long run. ${ }^{9}{ }^{10}$ In our study three SRWU schedules were evaluated in 16 children with asthma, and the efficiency of the best of these SRWU was then examined in a further series of 30 asthmatic children.

\section{Methods}

The tests were conducted outdoors in a centre for the treatment of asthmatic children in the Pyrenees mountains at an altitude of $1800 \mathrm{~m}$. This centre is dedicated to improving the asthmatic status of the children through physical activity in a non-polluted environment. Ambient mean (SD) temperature was $4(5)^{\circ} \mathrm{C}$ with a low humidity $(1.5(0.5) \mathrm{mm} \mathrm{Hg})$.

\section{FIRST STUDY}

The first study aimed at selecting an optimum SRWU schedule. Sixteen children, aged 11 (2) years, with post-exercise asthma were included. All were tested in the laboratory and showed a fall in $\mathrm{FEV}_{1}$ of at least $15 \%$ after completion of a short 7 minute maximal exercise performed on an ergometric bicycle. Among them, five had a severe form of asthma, six a moderate form, and five a light form. The severity of the disease was classified according to the treatment (light: less than one $\beta_{2}$ agonist inhalation a day; moderate: one $\beta_{2}$ agonist inhalation a day, on average, with or without corticosteroids; severe: several $\beta_{2}$ agonist inhalations a day with corticosteroid inhalation).

On the first day the children were asked to run as fast as possible for a maximum of 7 minutes on the outdoor track of a stadium (EX1). The distance and the time required to run this distance were recorded. On three other different days each child performed an exercise similar to EX1 (designated EX2) 10 minutes after recovery from an SRWU schedule, with three different schedules of SRWU. For each child the characteristics of the SRWU periods were derived from the values of EX1 variables. The basic schedule of the SRWU was the same: two series of five short runs, each of $7.5 \% \mathrm{EX} 1$ distance with a recovery of $1 \mathrm{~min} 30 \mathrm{~s}$ between runs and of 5 minutes between the two series of runs (fig 1). The SRWU schedules differed in the speed of the runs: $100 \%, 120 \%$, and $130 \%$ of EX1 (that is, 31, 26, and 24 seconds) for SRWU 1, 2, and 3, respectively. Distances were indicated by posts on the track and the children were cheered to run more or less rapidly to reach the pre-set rate.

The SRWU schedules were carried out in a random order and were all performed during the mid-day period. The children did not received any medication 12 hours before the test. Maximal expiratory peak flows were measured with the mini Wright peak flow 


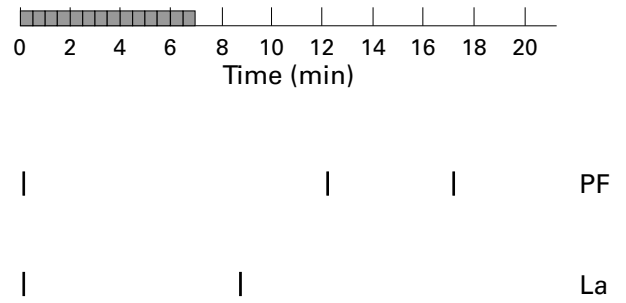

Second day: SRWU + EX2

\begin{tabular}{|c|c|c|c|c|c|c|c|c|c|c|c|c|c|c|c|c|c|c|c|c|c|c|c|c|}
\hline \multicolumn{5}{|c|}{ SRWU } & \multicolumn{3}{|c|}{$\mathrm{R} 1$} & \multicolumn{3}{|c|}{ SRWU } & \multicolumn{4}{|c|}{$\mathrm{R} 2$} & \multicolumn{2}{|l|}{ R3 } & \multicolumn{3}{|c|}{ EX2 } & & \multirow[b]{2}{*}{1} & \multirow[b]{2}{*}{$\perp$} & \multirow[b]{2}{*}{ | } & \multirow[b]{2}{*}{$\perp$} \\
\hline$\square$ & $\square$ & $\Pi$ & $\sqcap$ & $\square$ & 1 & $\perp$ & $\Gamma$ & $\square$ & $\Gamma$ & $\sqcap$ & $\Pi$ & $\perp$ & $\perp$ & 1 & 1 & & 111 & $\square 111$ & 110 & 1 & & & & \\
\hline 0 & 2 & 4 & 6 & 8 & 10 & 12 & 14 & 16 & 18 & 20 & 22 & 24 & 26 & 28 & 30 & 32 & 34 & 36 & 38 & 40 & 42 & 44 & 46 & 48 \\
\hline & & & & & & & & & & & & ime & (min) & & & & & & & & & & & \\
\hline 1 & [ & [] & [ & 1 & ] & [ & ] & & | & & | & | & & [ & & I & & & & & & I & & [ $\mathrm{PF}$ \\
\hline
\end{tabular}

Figure 1 Exercise procedure for EX1 (a 7 minute run alone) and EX2 ( a 7 minute run after short, repeated warm up schedules (SRWU)). The guide marks show the time of measurements of peak flow and lactate (La).

meter (Clement Clarke International, UK) before exercise, once after each run, between the two series of short runs (R1), 5 and 10 minutes after completion of the SRWU (R2, $\mathrm{R} 3)$, and 5 and 10 minutes during the recovery period after EX2. The best of three values of peak flow for each series was recorded. Blood lactate concentrations were measured before exercise and 2 minutes after completion of EX1, SRWU, and EX2 using a Microzym analyser (Setric genie industriel, France).

\section{SECOND STUDY}

The second study aimed at validating the effect of the chosen SRWU schedule on a larger group. Thirty children ( 21 boys, 9 girls) with post-exercise asthma, having light (4/30), moderate (18/30), or severe forms (8/30) of the disease, were included; their ages ranged from 8 to 15 years (mean 12 years). Ten of the 16 children from the first study were included in the second study. All children had an unambiguous diagnosis of asthma with no associated disease. All were exercise tested in the laboratory and all showed a fall in $\mathrm{FEV}_{1}>15 \%$ after completion of a 7 minute maximal exercise period on an ergometric bicycle.

For each child the experiments were completed in two consecutive days. All tests were performed during the mid-day period. All short acting bronchodilating agents were stopped for at least 12 hours before the tests. None of the children received long acting $\beta_{2}$ agonists, though most of the children with moderate to severe forms of asthma received corticosteroids by inhalation. Before exercise all children had a peak flow above $70 \%$ of the normal mean value. None had differences between initial peak flow greater than $20 \%$.

PROCEDURE (FIG 1)

On the first day the children were asked to run as fast as possible for a maximum of 7 minutes on the outdoor track of a stadium (EX1). The distance and the time required to run this distance were recorded. The characteristics of the SRWU period for each child were derived from the values of these variables.

On the second day the children performed an SRWU2 and a second exercise (EX2) similar to EX1, after 10 minutes of recovery. The SRWU2 consisted of two series of five short runs, each of $7.5 \%$ EX1 distance, at a speed slightly above that of EX1 (120\%)-that is, 26 seconds. SRWU2 was performed with a recovery of 1 minute and 30 seconds between the runs and 5 minutes between the two series of runs. After completion of the SRWU2 the children recovered for 10 minutes and then performed EX2. Peak flow and blood lactate measurements were made at the same time as in the first study; lactates: before exercise and 2

Table 1 Lactate concentrations and peak flow percentage change after SRWU1, 2, and 3 and distances run in the respective exercise tests. Rest peak flows are expressed as a percentage of the predicted peak flow

\begin{tabular}{lccccccccc}
\hline & Rest & SRWU1 & \multicolumn{1}{l}{ EX2 } & Rest & \multicolumn{1}{l}{ SRWU2 } & \multicolumn{1}{l}{ EX2 } & Rest & SRWU3 & EX2 \\
\hline Lactate (mmol/1) & $3.1(0.9)$ & $2.9(1)$ & $7.3(2.4)$ & $3.1(0.8)$ & $3.8(1.3)$ & $9.1(3.3)$ & $2.7(0.9)$ & $4.3(2)$ & $8.2(3.8)$ \\
Peak flow (\%) & $99(15)$ & $-5.4(7.5)$ & $-30.5(20)$ & $103.2(12)$ & $-6.8(10)$ & $-24.9(25)$ & $98.3(12)$ & $-10.1(10)$ & $-22.4(21)$ \\
Distance (m) & & & $1116(204)$ & & & $1133(223)$ & & 1137 & $(225)$ \\
\hline
\end{tabular}

SRWU 1, 2, and 3 = short, repeated warm up schedules; EX2 = a seven minute run after an SRWU. 


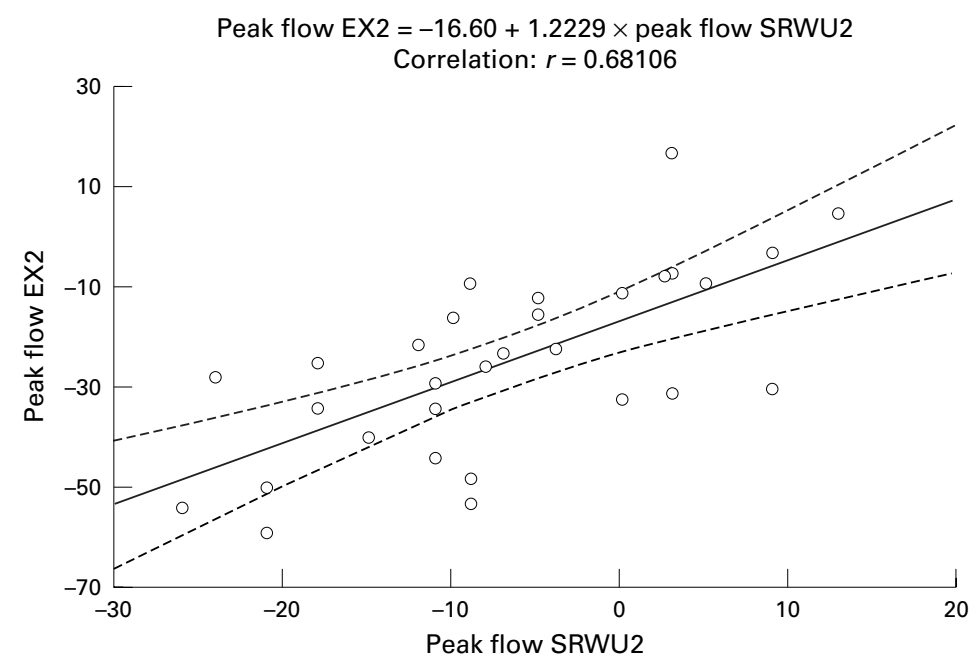

Figure 2 Percentage change in peak flow after EX2 as a function of the percentage change in peak flow after SRWU2. Continuous line = regression line; dotted lines $=95 \%$ confidence interval.

minutes after completion of EX1, SRWU, and EX2; peak flow: once after each run, between the two series of short runs (R1), 5 and 10 minutes after completion of SRWU2 (R2, R3), and 5 and 10 minutes during the recovery period after EX2.

\section{STATISTICAL ANALYSIS}

Data are expressed as means with standard deviation. Peak flow changes after SRWU, EX1, or EX2 were calculated as suggested by Meslier $e t a l^{11}$ as (initial peak flow - final peak flow)/reference peak flow, where reference peak flow is the predicted value as a function of height. ${ }^{12}$ Analysis of variance for repeated measures was used to compare the individual data and data between groups respectively. The planned comparison (LSD) test and Duncan's test were used for post-hoc test. Differences were considered significant at $\mathrm{p}<0.05$. Correlation was evaluated by Pearson's coefficient.

\section{Results}

The mean (SD) distance run during the first EX1 by the first group of 16 children was 1071 (256) $\mathrm{m}$ and the mean fall in peak flow was $-40.6(20) \%$, ranging from $-20.5 \%$ to $-79 \%$. The lactate concentrations were $2.9(0.8)$ $\mathrm{mmol} / \mathrm{l}$ and $6.9(2.5) \mathrm{mmol} / \mathrm{l}$ before and after exercise respectively. Table 1 lists the lactate concentrations and peak flow after SRWU 1, 2, and 3 and their respective EX2. An analysis of variance showed no differences in peak flow variations between the SRWU1, 2, and 3 or after EX2. Lactate concentrations were significantly increased compared with initial values after SRWU2 and SRWU3 but not after SRWU1. There was a significant difference between the increase in lactate concentrations after SRWU1 and both SRWU2 $(p=0.002)$ and SRWU3 $(p=0.0004)$, as there was no difference between EX2 figures whichever SRWU was followed.

The average distance run during EX1 by the second group of 30 children was 1170 (142) m (range $950 \mathrm{~m}$ to $1420 \mathrm{~m}$ ). Two children ran for fewer than 7 minutes $(6 \mathrm{~min} 50 \mathrm{~s}$ and $5 \min 35$ s). Baseline values of peak flow were same for the two days of the study: EX1 or SRWU2 + EX2. EX1 was followed by a mean fall in peak flow of $-37(14.5) \%$ (range $-15 \%$ to $-77 \%$; from 341 (72) $1 / \mathrm{min}$ to 210 (55) $1 / \mathrm{min}$ ). After SRWU2 the mean fall in peak flow was -6.9 (10.2)\% and after EX2 it was -25 (18.2)\% (range $+17 \%$ to $-59 \%$, from 342 (76) $1 / \mathrm{min}$ to 253 (78) $1 / \mathrm{min}$ ). Peak flow decreases after exercise were significantly less after EX 2 than EX1 $(\mathrm{p}=0.0002)$, but lactate concentrations were little changed $(4.83 \mathrm{mmol} / 1 \approx 5.21 \mathrm{mmol} / \mathrm{l})$. The percentage fall in peak flow after EX2 was correlated with that after SRWU2 $(r=0.68$, $\mathrm{p}<0.001, \mathrm{n}=30$ ) (fig 2). There was less correlation between the percentage fall in peak flow after EX2 and the percentage fall in peak flow after $\operatorname{EX1}(r=0.56, \mathrm{p}<0.01, \mathrm{n}=30)$.

Three subgroups of children were defined according to their response to the SRWU2that is, to their alteration in peak flow at R2 or R3 (the worst alteration in peak flow during either R2 or R3 was maintained). During the warm up period 10/30 children showed a progressive increase in peak flow or no change in peak flow (group G1), 14/30 had a fall in peak flow of $<15 \%$ on completion of the SRWU2 (group G2), while the remaining children $(6 / 30)$ showed a fall in peak flow of $>15 \%$ (group G3). There was no difference between the three groups for peak flow after EX1, though the decrease in peak flow in G1 was smaller than those in G2 and G3 after EX2. After EX2 the fall in peak flow was significantly less than that after EX1 ( $<<0.01)$, except in G3 (table 2). Figure 3 shows that the children exhibiting a fall in peak flow during SRWU2 were less protected than the others. Lactate concentrations did not vary between the three subgroups either after SRWU2 or after EX1. Nevertheless, SRWU2 significantly increased lactate concentration, but significantly less than EX1 and EX2 ( $p=0.0001)$ (table 2).

Table 2 Percentage changes in peak flow and increases in lactate concentrations (d.Lact $=$ difference between post-exercise conditions and rest) after EX1, $S R W U 2$, and EX2. Distances run during EX1 and EX2

\begin{tabular}{|c|c|c|c|c|c|c|c|c|}
\hline & \multicolumn{3}{|l|}{ Peak flow } & \multicolumn{3}{|l|}{ d. Lact } & \multicolumn{2}{|c|}{ Distance } \\
\hline & EX1 (\%) & SRWU2 (\%) & $E X 2(\%)$ & EX1 (mmolli) & $\begin{array}{l}\text { SRWUU2 } \\
\text { (mmollll) }\end{array}$ & EX2 (mmolll) & $E X 1(m)$ & $E X 2(m)$ \\
\hline $\mathrm{G} 1(\mathrm{n}=10)$ & $-31.8(12)$ & $+4.75(4.2)$ & $-10.85(16)^{\star}$ & $+5.64(2.8)$ & $+1.43(1.21)$ & $+5.91(1.62)$ & $1234(142)$ & $1301(162)$ \\
\hline $\mathrm{G} 2(\mathrm{n}=14)$ & $-37.4(13)$ & $-9(3)$ & $-28(14)^{\star}$ & $+4.69(1.82)$ & $+0.93(1.38)$ & $+4.95(2.16)$ & $1142.85(193)$ & $1200(191)$ \\
\hline $\mathrm{G} 3(\mathrm{n}=6)$ & $-44.6(19.5)$ & $-21.3(3.2)$ & -41.6 (14) NS & $+3.84(2.7)$ & $+1.34(1.02)$ & $+4.65(1.79)$ & 1136.66 (112) & $1156.66(119)$ \\
\hline
\end{tabular}

$\mathrm{EX} 1$ = a seven minute run alone; EX2 = a seven minute run after an SRWU; SRWU = short, repeated warm up schedules; G1 = increase in peak flow; G2 = <15\% fall in peak flow; $\mathrm{G} 3=>15 \%$ fall in peak flow.

${ }^{\star} \mathrm{p}<0.01$ peak flow EX2 $v$ peak flow EX1. 


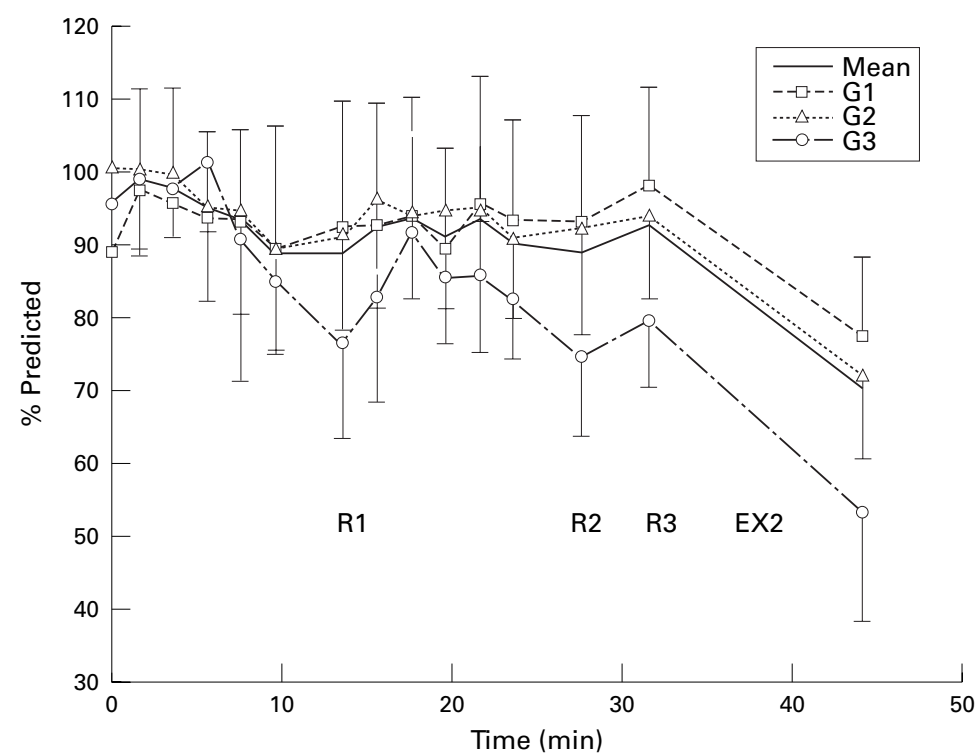

Figure 3 Percentage of the predicted peak flow during the SRWU2 and after EX2 in the three subgroups of children. R1 is the recovery period between the two series of short runs of the SRWU2. R2 and R3 are the alterations in peak flow during recovery after the SRWU2 (5 and 10 minutes after the end). EX2 is the final change in peak flow after the 7 minute exercise.

\section{Discussion}

The main finding of this study was that a well tolerated period of SRWU tailored to the ability of each individual reduced the severity of post-exercise asthma in most children. Some points should be noted before discussion of the main finding. Measurements were made at altitude, though the peak flow meter was calibrated for sea level measurement. However, the underestimation in peak flow measured with the mini Wright below 2000 metres is around $4 \% .{ }^{13}$ The recorded values of peak flow were not corrected for this error as all values were collected under the same conditions. The thermo-hygrometric conditions might have altered the response of the children to exercise, though all measurements were made in cold dry conditions, which are known to enhance post-exercise bronchospasm. A free run during 7 minutes in a cold dry air environment (EX1) was selected as this type of exercise has been reported to be the most bronchospastic. ${ }^{14-16}$

There was no relation between the lactate concentration and the fall in peak flow after EX1. Moreover the increase in lactate concentration after SRWU was $1-2 \mathrm{mmol} / 1$, around $20 \%$ of that after EX1 or EX2. Nevertheless, this increase was small and unlikely to produce a strong acidotic ventilatory drive. Indeed, although SRWU represented the same amount of work as $\mathrm{EX} 1(75 \%$ in duration at a speed $120 \%$ EX1), lactate could be metabolised during the recovery periods between the runs. On the other hand, EX2 lactate concentrations were slightly but not significantly increased compared with those found after EX1. This increase was attributed to the slight increase in overall distance run during EX2.

The choice of SRWU schedule was based on the results of Schnall and Landau, ${ }^{9}$ who showed that seven consecutive 30 second runs with 2 min $30 \mathrm{~s}$ breaks reduced the post- exercise bronchospasm of a 6 minute maximal exercise on a treadmill. However, in their study the fall in peak flow without SRWU was modest $(-22.8(8.1) \%)$ and was conducted with a small sample of combined adults and children. Moreover, McKenzie et $a l^{17}$ reported that an intermittent warm up provided even lower protection, without statistical significance, to post-exercise bronchospasm. They showed that eight consecutive 30 second runs ( $1.5 \mathrm{~min}$ rest) at an intensity equivalent to $100 \% \mathrm{VO}_{2} \mathrm{MAX}$, performed before a 6 minute treadmill exercise test, reduced the fall in $\mathrm{FEV}_{1}$ (forced expiratory volume in one second) induced by this test by only $6 \%$. Therefore it appeared that the effect of intermittent exercise on post-exercise bronchospasm had to be reinvestigated.

Our study aimed at re-examining the effect of an intermittent warm up in a larger series of asthmatic children and determining an optimum speed and duration for the short runs. SRWU1 was found to be less protective against bronchospasm than the other SRWU schedules. The running rate in SRWU1 was very slow and the lactate concentration did not differ from that at rest. SRWU2 and SRWU3 produced comparable reductions in post-exercise bronchospasm, and significant increases in lactate concentrations $(p=0.027$ and 0.005 respectively). Despite the lack of statistical differences between these two schedules, SRWU3 was reported to be more strenuous by the children. This feeling is supported by the slightly greater lactate concentration (table 1). Therefore, SRWU3 may be of more interest for children wanting a harder training regimen, as it was as protective as SRWU2.

Most of the children included in the second study were partially protected from bronchospasm by SRWU2. Indeed 24 out of 30 showed a smaller decrease in peak flow after EX2 than after EX1 (gain $17.39(11.5) \%$ ) as 10 of then showed a gain of $20.95 \%$ in peak flow after SRWU2 (G1) and 14 showed a gain of $9.71 \%$ in peak flow (G2) (table 2). The three subgroups showed no difference in their percentage fall in peak flow after EX1. However, the three groups responded differently to SRWU2, suggesting that in some children (G1) SRWU2 had a bronchodilator-like effect, protecting the children from severe fall in peak flow after EX2. This is supported by the fact that in others, SRWU2 had a slight bronchoconstrictor effect amplified after EX2. The sensitivity to the warm up schedule was thus not closely related to the severity of the bronchoconstriction occurring during a previous exercise (EX1), but there was a high correlation between the fall in peak flow after SRWU2 and that after EX2 $(r=0.68)$. This suggests that children with a fall in peak flow of $>15 \%$ after the warm up schedule were likely to develop a severe bronchospasm after a long run, whereas those who bronchodilate or had no change in peak flow during SRWU were more likely to be protected against a severe bronchoconstriction. Haas et $a l^{18}$ noted that after 36 sessions of aerobic training, asthmatic subjects had a greater bronchodilatation during exercise followed by a reduction of post- 
exercise bronchoconstriction. Moreover, the post-exercise bronchospasm severity was in part related to the magnitude of bronchodilatation during exercise. Overall, our data and those of Haas et $a l^{18}$ suggest that the severity of post-exercise bronchospasm is related both to the status of the bronchi during exercise and after a warm up procedure.

The mechanism responsible for protection against a subsequent exercise challenge remains uncertain, but the finding that indomethacin abolishes the refractory period suggests that the protection afforded by repeated exercise might be due to the release of inhibitory prostaglandins. ${ }^{19}{ }^{20}$ We surmised that inhibitory prostaglandins were released during SRWU and limited the exercise-induced bronchoconstriction, although this needs to be confirmed by giving indomethacin during the warm up procedure.

What might be the benefit of SRWU compared with the anti-asthmatic drugs before exercise? $\beta 2$ Agonists, nedocromil and sodium cromoglycate, have been shown to prevent post-exercise bronchospasm. ${ }^{21}{ }^{22}$ However, a warm up period, commonly recommended before sport or physical activity, and SRWU is a particular form of warm up, might avoid the need for medication before exercise, at least in some children with asthma. We also think it beneficial for children to be able to take exercise or play sports without or with minimal medication as it helps them feel less dependent on it.

In conclusion, a personalised warm up schedule was found to attenuate the decrease in peak flow for most of the children in our series $(80 \%)$, thus reducing subsequent post-exercise deep bronchoconstriction. As a warm up is always advised before performing a physical activity, it might be worthwhile selecting those children with asthma who are well protected by SRWU (G1) and training them to carry out this particular warm up procedure to enable them to exercise without medication. Further studies will be needed to define the effect of the association of medication and SRWU in the G2 and G3 groups. It seems likely that the children in these groups benefiting from SRWU would need less medication to be protected against exercise-induced bronchoconstriction.

We thank Professor A Taytard for his help with the initial experimental design, and Dr Calvayrac for recruiting the children in this study.
Contributors

Hervé Guénard discussed core ideas, participated in documentation, analysis, interpretation, and writing of the paper. Claire de Bisschop, the principal investigator of the SRWU study, designed the protocol and participated in data documentation, data collection, analysis, interpretation, and writing of the paper. Philippe Desnot designed and performed the experiments, and contributed to the paper. Jean Vergeret initiated the research, coordinated the formulation of the primary study hypothesis, and participated in the analysis and interpretation of the data.

1 Katz RM. Asthma and sports. Ann Allergy 1983;51:153-60. 2 Strunk RC, Rubin D, Kelly L, et al. Determination of fitness in children with asthma. Am f Dis Child 1988;142:940-4.

3 Harsha D. The benefits of physical activity in childhood. Am f Med Sci 1995;310(suppl 1): S109-13.

4 Strunk RC, Mrazeck DA, Fukuara JT, et al. Cardiovascular fitness in children with asthma correlates with psychologic functioning of the child. Pediatrics 1989;84:460-4.

5 Engstrom I, Fallstrom K, Karlberg E, et al. Psychological and respiratory physiological effect of a physical exercise program on boys with severe asthma. Acta Paediatr Scand 1991;80:1058-65.

6 Ries AL. The importance of exercise in pulmonary rehabilitation. Clin Chest Med 1994;15:327-37.

7 Bundgaard A, Ingeman-Hansen T, Halkjaer-Kristensen J, et al. Short term physical training in bronchial asthma. $B r \mathcal{F}$ Dis Chest 1983;77:147-52.

8 Morton AR, Fitch KD, Davis T. The effect of warm-up on exercise-induced asthma. Ann Allergy 1979;42: 257-60.

9 Schnall RP, Landau LI. Protective effect of repeated short sprints in exercise-induced asthma. Thorax 1980;35:82832.

10 Vergeret J, de Bisschop C, Simon G, et al. Recherche et évaluation sur le terrain des bénéfices d'un échauffement personnalisé dans la prévention du bronchospasme postexercise. Rev Mal Respir 1986;3:167.

11 Meslier N, Racineux JL, Six P, et al. Diagnostic value of reversibility of chronic airway obstruction to separate asthma from chronic bronchitis : a statistical approach. Eur Respir f 1989;2:497-505.

12 Godfrey S, Kamburoff PL, Nairn JR. Spirometry, lung volumes and airway resistance in normal children aged 5 to 18 years. Br F Dis Chest 1970;64:15-24.

13 Guénard $\mathrm{H}$, de Bisschop C. Débits ventilatoires maximaux en altitude. Sciences et Sport 1989;4:137-43.

14 Anderson SD, Schoeffel RE. The importance of standardising exercise test in the evaluation of asthmatic children. In: Oseid S, Edwards AM, eds. The asthmatic child in play and sport. London: Pitman, 1983:145-57.

15 Cropp GJA. The exercise bronchoprovocation test: standardization of procedures and evaluation of response. $\mathcal{F}$ Allergy Clin Immunol 1979;64:627-33.

16 Custovic A, Arifhodzic N, Robinson A, et al. Exercise testing revisited. Chest 1994;105:1127-32.

17 McKenzie DC, Mc Luckie SL, Stirling DR. The protective effects of continuous and interval exercise in athletes with exercise-induced asthma. Med Sci Sports Exerc 1994;26: 951-6.

18 Haas F, Pasierki S, Levine N, et al. Effect of aerobic training on forced expiratory airflow in exercising asthmatic human. 7 Appl Physiol 1987;63:1230-5.

19 O'Byrne PM, Jones GL. The effect of indomethacin on exercise induced bronchoconstriction and refractoriness after exercise. Am Rev Respir Dis 1986;134:69-72.

20 Margolskee DJ, Bigby BG, Boushey HA. Indomethacin blocks airway tolerance to repetitive exercise but not to eucapnic hyperpnea in asthmatic subjects. Am Rev Respir Dis $1988 ; 137: 842-6$.

$21 \mathrm{McFadden}$ ER, Gilbert IA. Exercise-induced asthma. $N$ Engl f Med 1994;330:1362-7.

22 Morton AR, Fitch KD. Asthmatic drugs and competitive sport. Sports Med 1992;14:228-42.

\section{Take home message}

This study provides some evidence of the favourable effect of short repeated warm ups on exercise-induced bronchoconstriction in children with asthma. 